\title{
Analysis of a commercial preparation of erythromycin estolates by tandem mass spectrometry and high performance liquid chromatography/electrospray ionization tandem mass spectrometry using an ion trap mass spectrometer
}

\author{
Chien-Chen Lai, Pei-Lun Tsai, Cheng Yu and Guor-Rong Her* \\ ${ }^{1}$ Department of Chemistry, National Taiwan University, Taipei, Taiwan, R.O.C.
}

\begin{abstract}
Because of the lack of a UV chromophore and their much smaller abundances in comparison with the major component, the minor components in erythromycin estolate preparations are difficult to analyze by high performance liquid chromatography ultraviolet (HPLC-UV). Tentative assignment of the major and minor components can be achieved with the combination of full scan and ZoomScan using an ion trap mass spectrometer. Tandem mass spectrometry (MS/MS) provided an effective method to quickly identify most components without chromatographic separation, and all the related compounds, except the isobaric pair ECE and PdMeEA, could be identified in this way. The best result was obtained by using liquid chromatography/tandem mass spectrometry (LC/MS/MS) operated in selected reaction monitoring mode. The major compound, the estolate of erythromycin A (EAE), and seven other minor components, could be separated and identified, with semiquantitative estimates of relative concentrations. Copyright $\mathbb{C} 2000$ John Wiley \& Sons, Ltd.
\end{abstract}

Received 29 November 1999; Revised 6 January 2000; Accepted 7 January 2000

Soon after the introduction of the antibiotic erythromycin in 1952, a number of esters were prepared and marketed. ${ }^{1,2}$ One of these derivatives is erythromycin propionate, which is often called erythromycin estolate, the lauryl sulfate salt of the 2 '-propionate ester. It has been formulated in both liquid and solid pharmaceutical forms and is still widely used. Esters of erythromycin are biologically inactive prodrugs and have to be hydrolyzed to produce antimicrobial activity.

The major component in erythromycin estolates is the estolate of erythromycin $\mathrm{A}$, and the minor components frequently observed in the product are the propionate esters of erythromycin B, C, E and F, erythromycin A, erythromycin $\mathrm{C}$, and $\mathrm{N}$-propionyl- $\mathrm{N}$-demethylerythromycin A (Fig. 1).

High performance liquid chromatography (HPLC) has been widely used for the analysis of the erythromycins and related substances. Methods based on HPLC/UV for purity control of bulk products and preparations have been reported. $^{3-5}$ However, the lack of a UV chromophore in the molecule represents a distinct challenge for the determination of erythromycin estolate and related substances. This inherent property hinders high sensitivity detection since UV absorption at low wavelengths is relatively poor. ${ }^{6}$

Because of its low detection limit, high specificity and,

*Correspondence to: G.-R. Her, Department of Chemistry, National Taiwan University, Taipei, Taiwan, R.O.C.

E-mail: grher@mail.ch.ntu.edu.tw more importantly, abundant structure information, mass spectrometry (MS) has been considered one of the ideal devices for drug analysis. Analysis of erythromycins by various conventional MS techniques have been reported. ${ }^{7-11}$ Recently, electrospray ionization has rapidly emerged as a very promising technique for the analysis of compounds with medium or high polarity. Similar to atmospheric pressure chemical ionization (APCI), electrospray ionization (ESI) produces ions at atmospheric pressure, but without the need for high temperature that could decompose labile compounds.

Concurrent with the development of soft ionization techniques has been the development of tandem mass spectrometry (MS/MS). MS/MS is known for its high specificity and the capability of structural elucidation. Recently, ESI-MS/MS and HPLC/ESI-MS/MS have been applied to the analysis of various erythromycins. ${ }^{8,10-12}$ These reports mainly focused on the analysis of the major erythromycin ${ }^{8,11}$ and its decomposition products. ${ }^{12}$ In this report, the major component, erythromycin estolate $\mathrm{A}$, as well as related minor substances, in a commercial preparation, were studied by MS, MS/MS and HPLC/MS/MS. The results of the analysis, and the relative merits and pitfalls of these techniques, are discussed.

\section{EXPERIMENTAL}

\section{Chemicals}

Erythromycin estolate capsules (Kindom, Taipei, Taiwan) were purchased from a local drug store. HPLC grade 


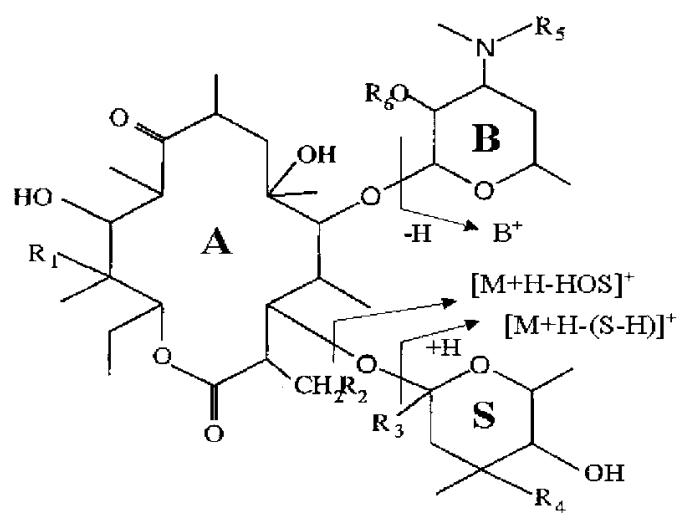

\begin{tabular}{|c|c|c|c|c|c|c|c|}
\hline Compound & $\mathrm{R}_{1}$ & $\mathrm{R}_{2}$ & $\mathrm{R}_{3}$ & $\mathrm{R}_{4}$ & $\mathrm{R}_{5}$ & $\mathrm{R}_{6}$ & M.W. \\
\hline Erythromycin A (EA) & $\mathrm{OH}$ & $\mathrm{H}$ & $\mathrm{H}$ & $\mathrm{OCH}_{3}$ & $\mathrm{CH}_{3}$ & $\mathrm{H}$ & 733 \\
\hline Erythromycin C (EC) & $\mathrm{OH}$ & $\mathrm{H}$ & $\mathrm{H}$ & $\mathrm{OHI}$ & $\mathrm{CH}_{3}$ & $\mathrm{H}$ & 719 \\
\hline Erythromycin A propionate (EAE) & $\mathrm{OH}$ & $\mathrm{H}$ & $\mathrm{H}$ & $\mathrm{OCH}_{3}$ & $\mathrm{CH}_{3}$ & $\mathrm{EtCO}$ & 789 \\
\hline Erythromycin B propionate (EBE) & $\mathrm{H}$ & $\mathrm{H}$ & $\mathrm{H}$ & $\mathrm{OCH}_{3}$ & $\mathrm{CH}_{3}$ & EtCO & 773 \\
\hline Erythromycin C propionate (ECE) & $\mathrm{OH}$ & $\mathrm{H}$ & II & OlI & $\mathrm{CH}_{3}$ & EtCO & 775 \\
\hline Erythromycin E propionate (EEE) & $\mathrm{OH}$ & $\mathrm{OH}$ & $\mathrm{H}$ & $\mathrm{OCH}_{3}$ & $\mathrm{CH}_{3}$ & $\mathrm{EtCO}$ & 805 \\
\hline Erythromycin F propionate (EFE) & $\mathrm{OH}$ & \multicolumn{2}{|c|}{-0} & $\mathrm{OCH}_{3}$ & $\mathrm{CH}_{3}$ & $\mathrm{EtCO}$ & 803 \\
\hline $\begin{array}{l}\text { N-propionyl-N-demethylerythromycin } \\
\text { A (PdMcEA) }\end{array}$ & $\mathrm{OH}$ & $\mathrm{H}$ & $\mathbf{H}$ & $\mathrm{OCH}_{3}$ & EtCO & $\mathrm{H}$ & 775 \\
\hline
\end{tabular}

Figure 1. Structures and integral molecular weights (truncated accurate masses) of erythromycin A propionate and related compounds.

acetonitrile was obtained from Lab-Scan Analytical Science (Labscan Ltd. Dublin, Ireland). Ammonium acetate $\left(\mathrm{NH}_{4} \mathrm{OAc}\right.$ ) was purchased from J. T. Baker (Phillipsburg, NJ, USA). Deionized (18M $\Omega$ ) water (Milli-Q water system, Millipore Inc., Bedford, MA, USA) was used in the preparation of the samples and buffer solution. Prior to use, the mobile phase was filtered through a $0.45 \mu \mathrm{m}$ membrane filter (Gelman Sciences, Michigan, USA).

\section{Preparation of samples}

In direct infusion experiments, the sample solution was prepared with $50 \%$ acetonitrile $(0.1 \%$ HOAc). For HPLC/ MS experiments, an erythromycin estolate sample solution (about $1000 \mathrm{ppm}$ ) was prepared in $1 \mathrm{~mL}$ of HPLC mobile phase. Both solutions were filtered with a $0.45 \mu \mathrm{m}$ filter and analyzed immediately after preparation.

\section{HPLC/UV system}

The chromatographic system consisted of two model LC10AD pumps (Shimadzu, Kyoto, Japan), a Dyna-Mix Plus mixer (Microtech Scientific, Inc., USA), a Rheodyne model 7125 injection valve (Rheodyne, USA) fitted with a $5 \mu \mathrm{L}$ loop, and a Rainin Dynamax UV-C UV detector set at $210 \mathrm{~nm}$ (Rainin, MA, USA). Chromatograms were recorded on a Pentium PC using an SISC PC Integrator software package (Scientific Information Service Corporation, Taipei, Taiwan).

HPLC analysis was performed on a reversed phase NovaPak $\mathrm{C}_{18}$ column $(2 \mathrm{~mm}$ i.d. $\times \mathrm{cm}$; Particle size, $3 \mu \mathrm{m}$, Waters Associates). A guard column (Nova-Pak $\mathrm{C}_{18}$ cartridge in a radial compression module) was used to prolong the life of the HPLC column. The mobile phase was

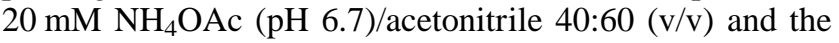
flow rate was $200 \mu \mathrm{L} / \mathrm{min}$.

\section{Mass spectrometry}

A Finnigan LCQ quadrupole ion trap mass spectrometer (Finnigan Corp., San Jose, CA, USA), equipped with a pneumatically assisted electrospray ionization source, was used. The mass spectrometer was operated in positive ion mode by applying a voltage of $4.5 \mathrm{kV}$ to the ESI needle. The temperature of the heated capillary in the ESI source was set at $200^{\circ} \mathrm{C}$. To avoid space charge effects, the number of ions stored in the trap was regulated by the automatic gain control, which was set at $4 \times 10^{7}$ ions for full scan mode, $2 \times 10^{7}$ for MS/MS mode, and $1 \times 10^{7}$ for ZoomScan mode. The flow rate of the sheath gas of nitrogen was set at 25 (arbitrary units). Helium was used as the damping gas at a pressure of $10^{-3}$ Torr. Voltages across the capillary and the octapole lenses were tuned by an automated procedure to maximize signal for the ion of interest.

In MS/MS analysis, typical values for the relative collision energy (peak-to-peak amplitude of the resonance excitation) ranged from 0.4 to $0.8 \mathrm{eV}$. Mass spectra collected in full-mass scan mode were obtained by scanning over the range $\mathrm{m} / \mathrm{z}, 150$ to 850 . The maximum ion collection time was $0.3 \mathrm{~s}$ for each step and 3 scans were added for each spectrum.

\section{RESULTS AND DISCUSSION}

\section{Full scan and ZoomScan analysis of erythromycins}

The structures and molecular weights of erythromycin propionate and related substances are shown in Fig. 1. In an initial electrospray MS study, the bulk sample of erythromycins was analyzed by infusing sample solution directly into the electrospray ion source. As mentioned earlier, the sample is the lauryl sulfate salt of the erythromycin $2^{\prime}$ propionate ester. The major ion detected in the negative ion ESI mass spectrum (data not shown) corresponds to the 


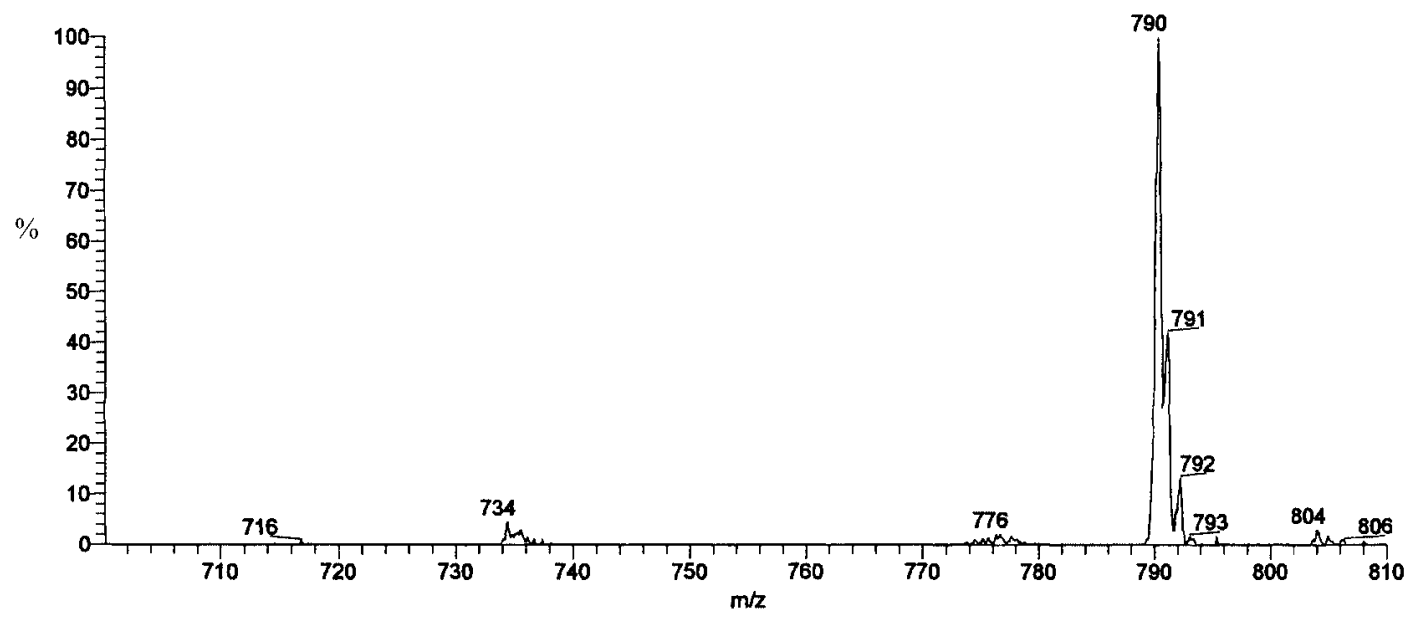

Figure 2. Positive ion ESI mass spectra of erythromycins.

lauryl sulfate anion. The positive ion electrospray mass spectrum is shown in Fig. 2. The base peak, observed at $\mathrm{m} / \mathrm{z}$ 790 , corresponds to protonated erythromycin A. In addition to the base peak, several low intensity ions were also observed. The ions at $\mathrm{m} / \mathrm{z}$ 734, 776 and 804 were assigned as the protonated molecules of erythromycin A (EA), erythromycin $C$ estolate (ECE) and erythromycin F estolate (EFE), respectively. The weak signal at $\mathrm{m} / \mathrm{z} .716$ was tentatively assigned as the $\left[\mathrm{M}+\mathrm{H}-\mathrm{H}_{2} \mathrm{O}\right]^{+}$fragment ion of EA.

The signal/noise and resolution in Fig. 2 are not good enough for a more conclusive assignment. To improve signal/noise as well as resolution, the ZoomScan facility was used. In our ion trap mass spectrometer, ZoomScan is a technique involving slower scan rates over a limited range with higher resolution. ZoomScans were performed at $1 / 20^{\text {th }}$ of the scan rate of the normal scan and using a preset window of $10 \mathrm{Th}$. The ZoomScan mass spectra of selected ions are shown in Fig. 3. The isotopic ratios calculated from Fig. 3(a) appear to be similar to the predicted isotopic ratios of $\operatorname{EAE}(\mathrm{M}: \mathrm{M}+1: \mathrm{M}+2: \mathrm{M}+3=100: 46: 13: 3)$. The abundance ratios of the $\mathrm{m} / \mathrm{z}, 734,735$ and 736 ions were also similar to the predicted values for EA (data not shown). In the ZoomScan mass spectrum of the $\mathrm{m} / \mathrm{z} 776$ ion, in addition to the $m / z 776$ ion which corresponds to the protonated molecules of ECE or PdMeEA, two other ions at $m / z, 774$ and 772 were detected (Fig. 3(b)). The ion at $m / z 774$ corresponds to the protonated molecule of EBE, and the $\mathrm{m} / \mathrm{z}$ 772 ion is most likely the $\left[\mathrm{M}+\mathrm{H}-\mathrm{H}_{2} \mathrm{O}\right]^{+}$fragment ion of (a)

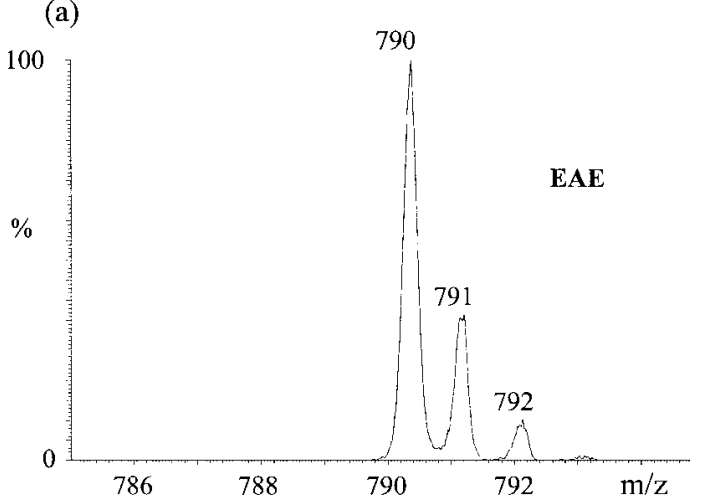

(c)

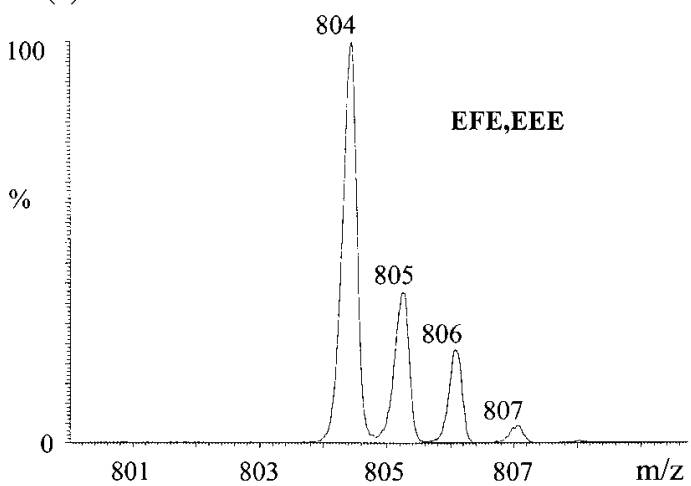

(b)

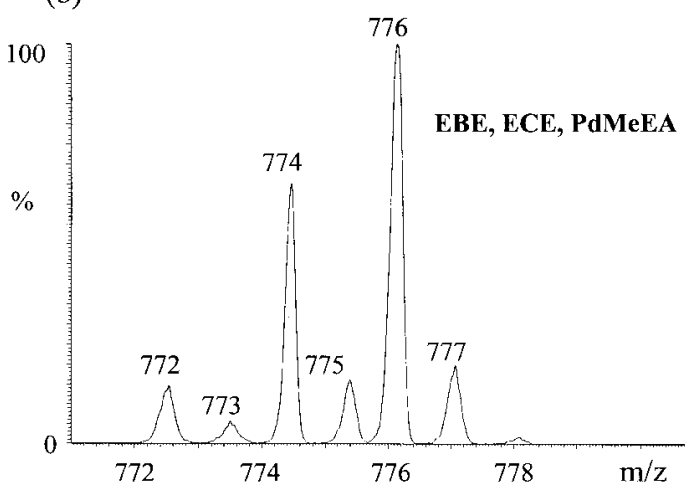

(d)

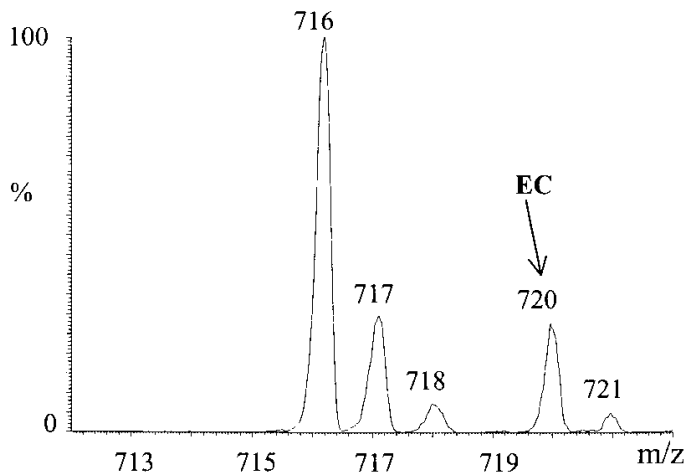

Figure 3. Positive ion ZoomScan ESI mass spectra of (a) $\mathrm{m} / \mathrm{z} 790$, (b) $\mathrm{m} / \mathrm{z} 776$, (c) $\mathrm{m} / \mathrm{z} 804$ and (d) $\mathrm{m} / \mathrm{z} 720$. 
(a)

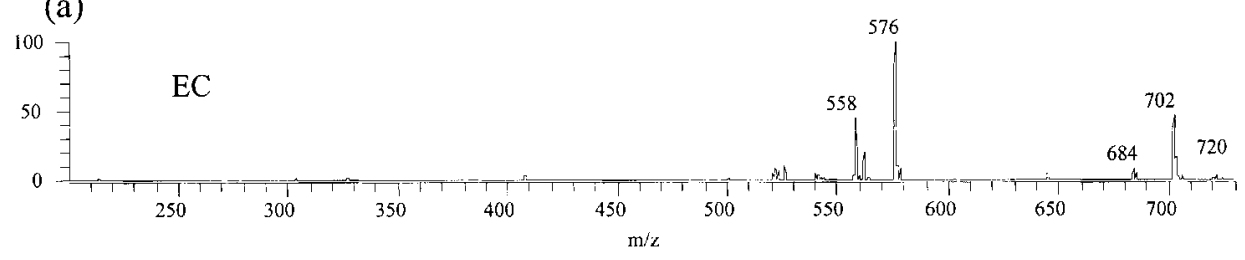

(b)

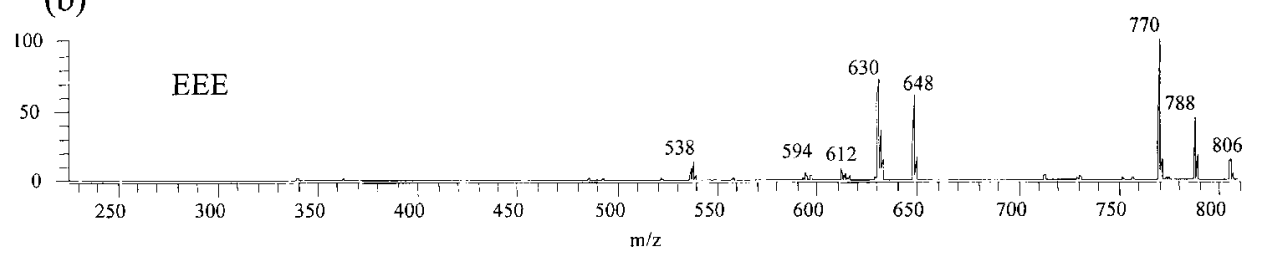

(c)
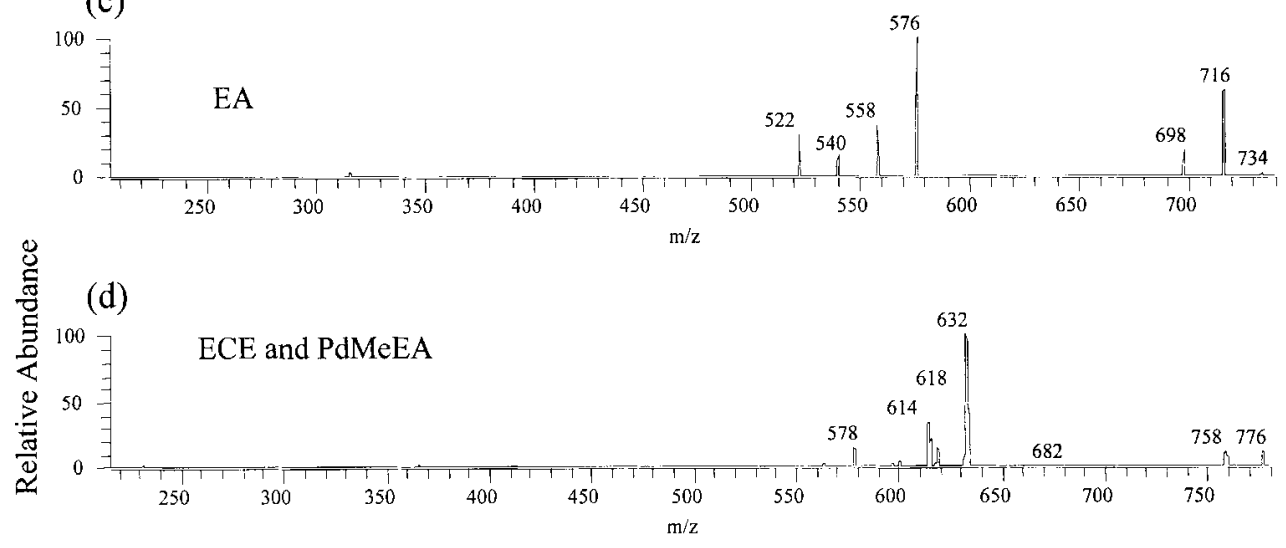

(e)

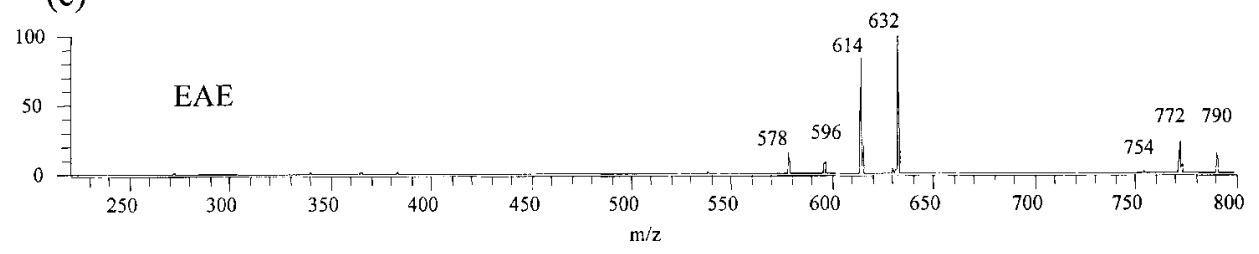

(f)

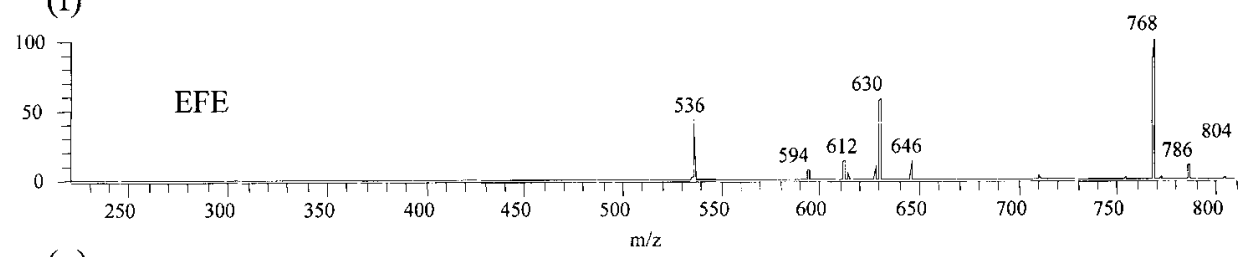

(g)

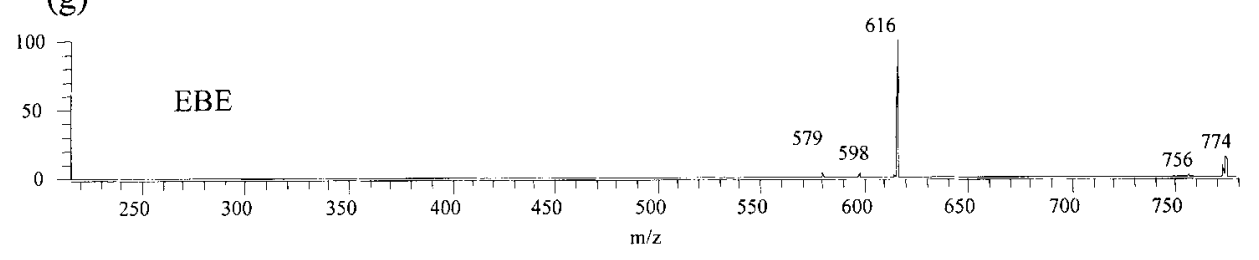

Figure 4. Positive ion ESI product ion mass spectra of (a) EC, (b) EEE, (c) EA, (d) ECE and PdMeEA, (e) EAE, (f) EFE and (g) EBE.

EAE. The relative abundance pattern of $\mathrm{m} / \mathrm{z} 804,805$ and 806 (Fig. 3(c)) was different from the theoretical values for EFE, in that the relative intensity of the $m / z 806[\mathrm{M}+2]$ ion (24\%) was significantly higher than the theoretical value (13\%). This discrepancy suggested the presence of a compound with a protonated molecule at $\mathrm{m} / \mathrm{z}$ 806. A possible candidate would be EEE. In Fig. 2, the weak signal at $m / z 716$ was tentatively assigned as the $\left[\mathrm{M}+\mathrm{H}-\mathrm{H}_{2} \mathrm{O}\right]^{+}$ fragment ion of EA. When ZoomScan was performed on the $\mathrm{m} / \mathrm{z} 716$ ion, an additional ion at $\mathrm{m} / \mathrm{z} 720$ was detected (Fig. $3(\mathrm{~d})$ ). This ion corresponds to the protonated molecule of erythromycin C (EC).

The ZoomScan approach provided useful information about the sample. However, even the specificity provided by ZoomScan was not high enough for a reliable assignment. Furthermore, ZoomScan also lacks the capability of differentiating ECE and PdMeEA because both compounds have the same nominal (integral) molecular weight. 


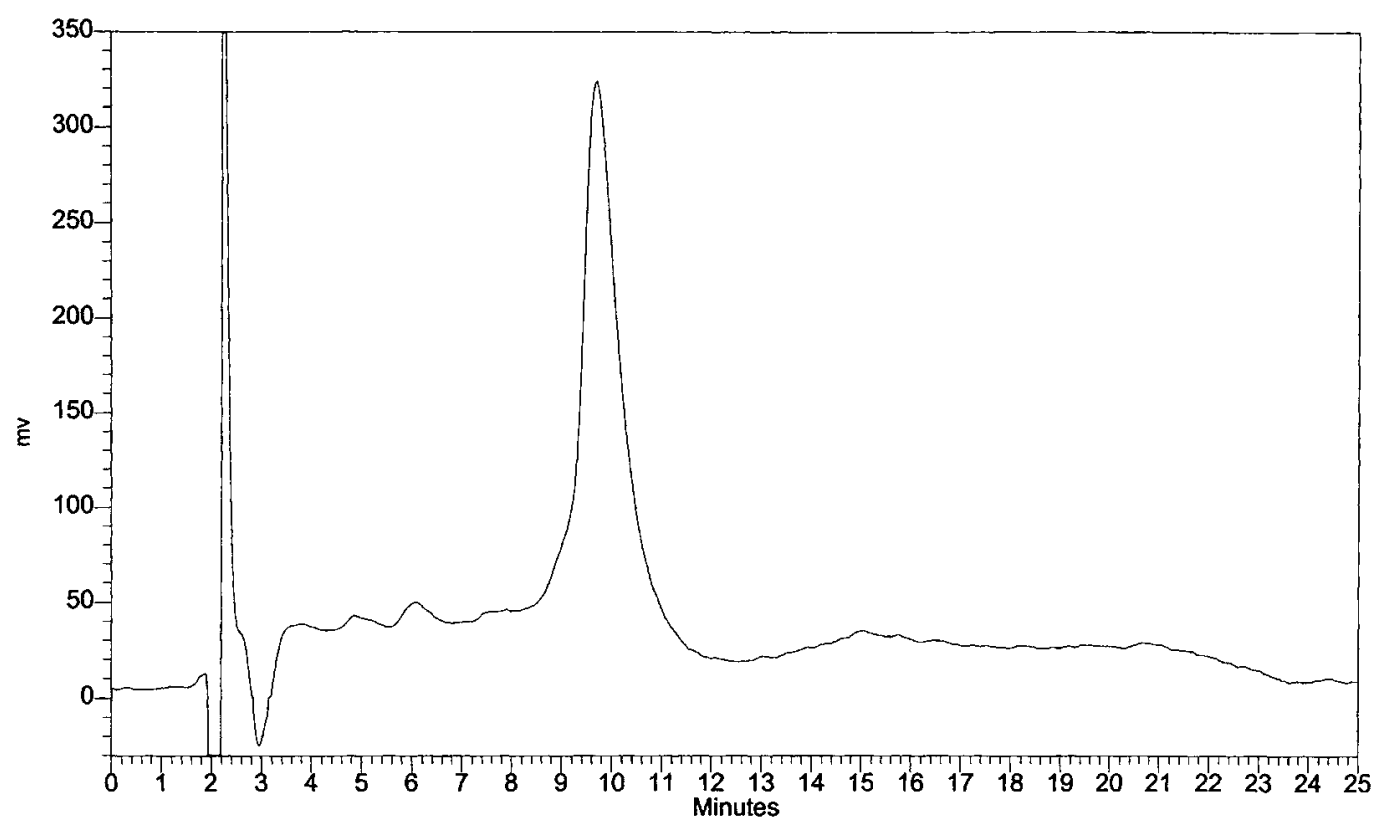

Figure 5. LC/UV chromatogram of a erythromycin estolate preparation. Mobile phase: $20 \mathrm{mM} \mathrm{NH}_{4} \mathrm{OAc}(\mathrm{pH}$ 6.7)/ acetonitrile 40:60 (v/v).

\section{MS/MS analysis of erythromycins}

Erythromycins are compromised of an amino sugar (B), a nitrogen-free sugar (S) and a 14-member ring aglycone portion (A). Because the proton is assumed to be located on the dimethylamino nitrogen, the major fragment ions observed for these compounds are $\mathrm{B}^{+},[\mathrm{M}+\mathrm{H}-(\mathrm{S}-\mathrm{H})]^{+}$ and $[\mathrm{M}+\mathrm{H}-\mathrm{HOS}]^{+}$(Fig. 1). The $\mathrm{m} / \mathrm{z}$ values of these fragment ions of each erythromycin are listed in Table 1. All the ions observed in ZoomScan were studied by MS/MS and the product ion spectra are shown in Fig. 4. The major ions observed were the $[\mathrm{M}+\mathrm{H}-(\mathrm{S}-\mathrm{H})]^{+}$and $[\mathrm{M}+\mathrm{H}-$ $\mathrm{HOS}^{+}$ions and their $\mathrm{m} / \mathrm{z}$ values were the same as those listed in Table 1 . The $\mathrm{B}^{+}$product ion was not detected because the ion trap could only scan to about $1 / 4$ of precursor ion mass. In addition to the major fragment ions, dehydrated ions were also observed. In Fig. 4(c), the ions detected at $\mathrm{m} / \mathrm{z} 716$ and 698 were the dehydrated $[\mathrm{M}+\mathrm{H}]^{+}$ ions of EA, and the $\mathrm{m} / \mathrm{z} 540$ and 522 ions were the dehydrated product ions of $[\mathrm{M}+\mathrm{H}-\mathrm{HOS}]^{+}$.

As mentioned earlier, ZoomScan is not capable of differentiating ECE and PdMeEA because both compounds have the same molecular weight. When the $m / z 776$ ion was subjected to analysis by MS/MS, it was interesting to see that product ions of both ECE and PdMeEA were detected

\begin{tabular}{lcccc}
\hline \multicolumn{5}{l}{ Table 1. $\boldsymbol{m} / \boldsymbol{z}$ values of the major fragment ions } \\
Compound & {$[\mathrm{M}+\mathrm{H}]^{+}$} & $\mathrm{B}^{+}$ & {$[\mathrm{M}+\mathrm{H}-(\mathrm{S}-\mathrm{H})]^{+}$} & {$[\mathrm{M}+\mathrm{H}-\mathrm{HOS}]^{+}$} \\
EA & 734 & 158 & 576 & 558 \\
EC & 720 & 158 & 576 & 558 \\
EAE & 790 & 214 & 632 & 614 \\
EBE & 774 & 214 & 616 & 598 \\
ECE & 776 & 214 & 632 & 614 \\
EEE & 806 & 214 & 648 & 630 \\
EFE & 804 & 214 & 646 & 630 \\
PdMeEA & 776 & 200 & 618 & 600 \\
\hline
\end{tabular}

$(\mathrm{m} / \mathrm{z}$ 632, 614 for ECE, $\mathrm{m} / \mathrm{z}$ 618, 600 for PdMeEA) (Fig. 4(d)). This result suggested that the sample contained both ECE and PdMeEA.

Although MS/MS does provide more specific information than ZoomScan, the product ion spectra were not easy to acquire for several minor components. Even when using a $1000 \mathrm{ppm}$ solution, the signals at $\mathrm{m} / \mathrm{z} 720,774$ and 806 were small and unstable, making an MS/MS study difficult. One possible explanation is that the much higher concentration of EAE in the solution suppresses the signal of the minor components.

\section{LC/MS and LC/MS/MS analysis of erythromycins}

In order to avoid the influence of EAE on ionization of the minor components, each component was separated by HPLC before analysis by MS. Among the published LC methods phosphate buffer was often used in the mobile phase, but unfortunately phosphate buffer is not suitable for analysis by ESI-MS. Ammonium acetate, a more volatile buffer, and acetonitrile, an organic modifier, were studied in the separation of erythromycin estolate by HPLC. It is known that ESI sensitivity can be improved if a mobile phase contains low-concentration volatile buffer and has a higher percentage of organic modifiers. Under the optimal conditions $\left(20 \mathrm{mM} \mathrm{NH} \mathrm{NH}_{4} \mathrm{OAc}, \mathrm{pH} 6.7,40: 60, \mathrm{v} / \mathrm{v}\right.$ acetonitrile), in addition to the major peak EAE, several small peaks were observed by LC/UV (Fig. 5). Because of the lack of a UV chromophore in the molecule, the signals of the minor components were quite weak. Since we do not have the authentic erythromycin standards, identification of the small peaks by retention time was not feasible.

When erythromycin estolates were analyzed by LC/ESIMS, the mass chromatograms shown in Fig. 6 were obtained. Peaks detected at 3.94, 4.01, 5.01, 5.97, 9.63, 10.30 , and $15.27 \mathrm{~min}$ correspond to $\mathrm{m} / \mathrm{z}, 720,806,734,776$, 790,804 and 774 ions, respectively. Based on these data, the elution order of erythromycins was assigned as EC, EEE, 

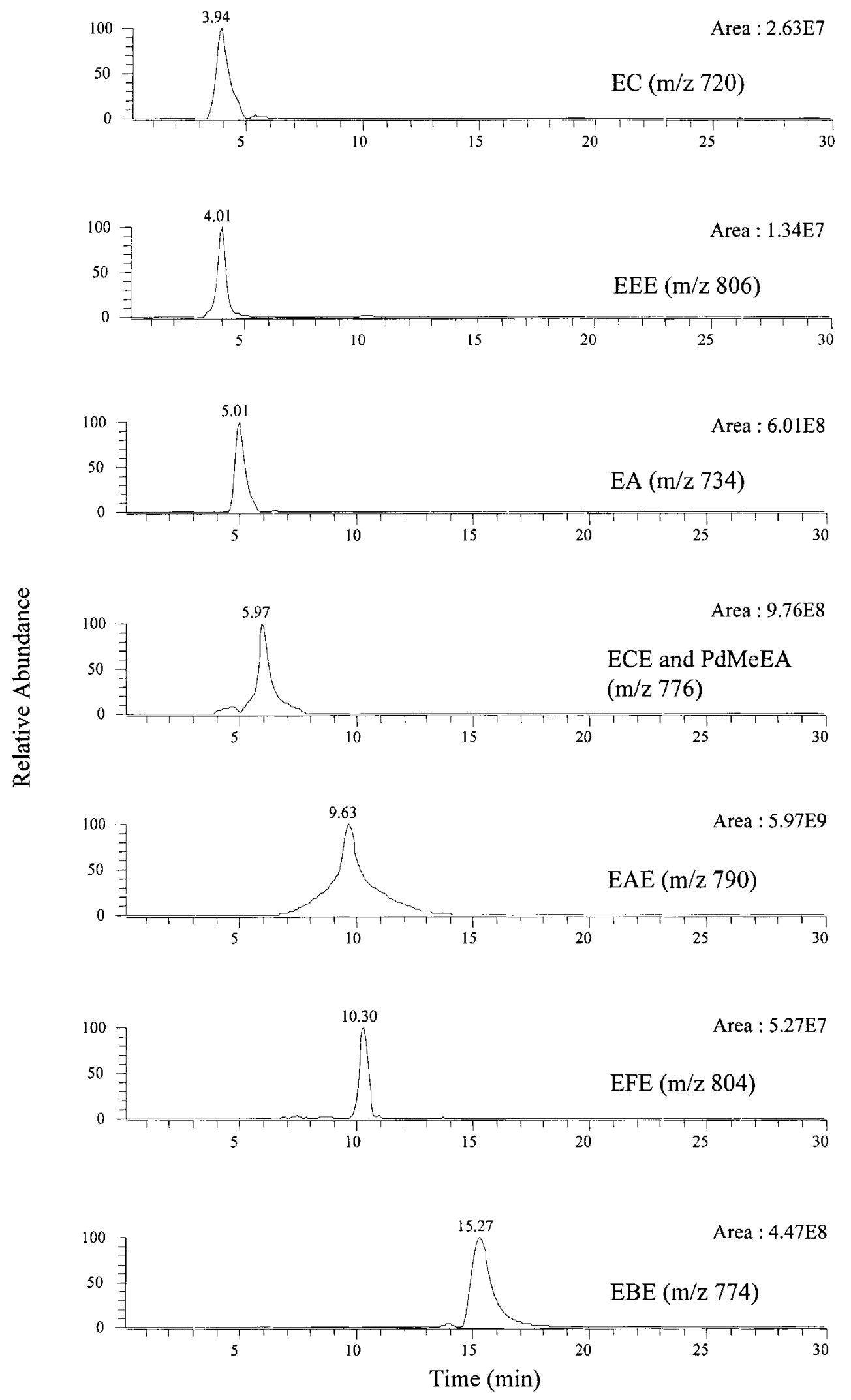

Figure 6. Mass chromatograms of erythromycins obtained under selected ion monitoring (SIM) operation. The chromatographic conditions were the same as in Fig. 5.

EA, ECE or PdMeEA, EAE, EFE and EBE. One advantage of using MS instead of UV as the detector is that baseline separation may not be needed for a clear identification. For example, EC could be identified even though it did not separate from EEE (Fig. 6). Another advantage of using MS instead of UV as the detector can be demonstrated by the detection of EFE by LC/MS but not by LC/UV. As suggested by the peak areas of EAE and EFE (Fig. 6), the concentration of EAE is much higher than that of EFE. The concentration difference was so great that the EFE peak was 

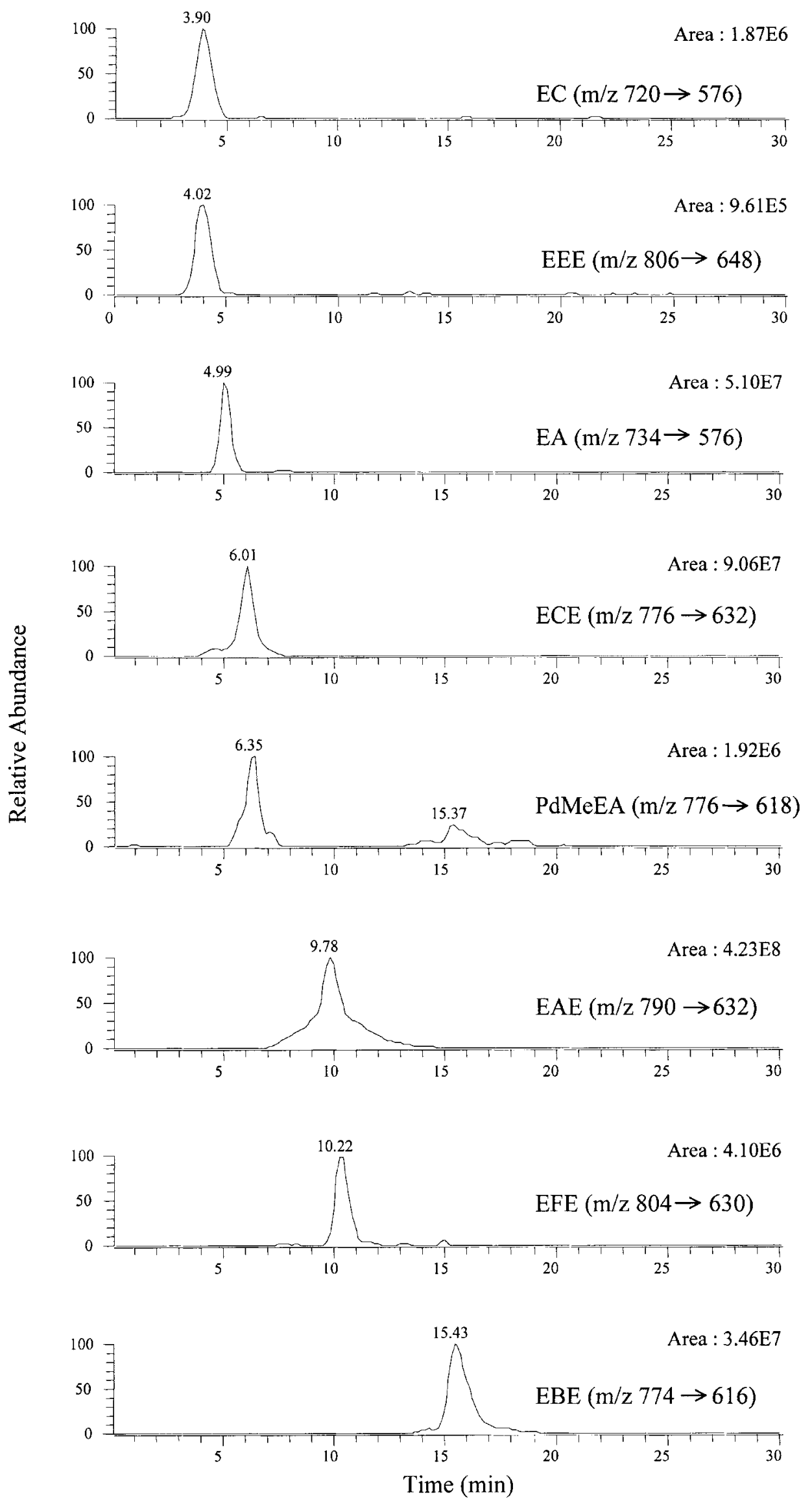

Figure 7. Mass chromatograms of erythromycins obtained under selected reaction monitoring (SRM) operation. The chromatographic conditions were the same as in Fig. 5. 
masked by the peak of EAE in Fig. 5 even though the difference in retention times between EFE and EAE was about $0.7 \mathrm{~min}$ (9.63 vs. $10.30 \mathrm{~min}$ ).

HPLC/MS is superior to MS/MS without chromatographic pre-separation in that relative concentrations of each compound can be estimated. The responses of different erythromycins are expected to be similar because the structures of these compounds are quite similar. Therefore, the ratios of peak areas can provide semiquantitative information about the relative concentrations in the sample, in the absence of pure standards of each analyte.

A peak corresponding to the $[\mathrm{M}+\mathrm{H}]^{+}$ions of ECE and PdMeEA was observed (5.97 $\mathrm{min})$. Without authentic standards, however, it was not possible to differentiate between ECE and PdMeEA because both compounds have the same nominal molecular weight.

To provide higher specificity for compound identification and also to solve the problem of ECE and PdMeEA, the sample was subjected to analysis by LC/MS/MS. The monitored reactions and SRM chromatograms for each erythromycin are shown in Fig. 7. Apart from the higher specificity provided by SRM, ECE and PdMeEA could be differentiated by SRM as shown in Fig. 7. Based on the peak areas, ECE was more abundant than PdMeEA, and accounted for most of the chromatographic peak observed in Fig. 6 at $5.97 \mathrm{~min}$. This is consistent with data from the product ion spectrum of $m / z, 776$ (Fig. 4(e)). The product ion of ECE $(\mathrm{m} / \mathrm{z}$ 632) had a higher abundance than that of PdMeEA $(\mathrm{m} / \mathrm{z}$ 618).

The small peak at $15.73 \mathrm{~min}$ in the $m / z$ 776-618 trace is due to the much more abundant EBE compound. In the MS/MS experiments, in order to detect the minor components, the peak width for both precursor and product ions was set to $3 \mathrm{Th}$, which was not adequate to resolve the $\mathrm{m} / \mathrm{z}$ $776 / 774$ precursor ions and the $618 / 616$ product ions.

\section{CONCLUSIONS}

In the analysis of an erythromycin estolate preparation, full scan along with ZoomScan provided tentative assignment about the major and the minor components in the sample. More reliable assignment could be achieved by MS/MS. Except for the two minor components with the same molecular weight, ECE and PdMeEA, MS/MS provided an effective method to identify the components directly without chromatographic separation. While MS/MS is very useful for fast qualitative analysis of most erythromycins, the best result was obtained by LC/MS/MS operated in selected reaction monitoring mode. The major compound, erythromycin A (EAE), and all other related minor components, present as the estolates but detected in positive ion electrospray as the protonated erythromycins, could be separated and identified. Furthermore, peak areas for each compound provided semiquantitative information about their relative concentrations in the sample.

\section{REFERENCES}

1. Mcguire LM, Bunch RL, Anderson RC, Boaz HE, Flynn EH, Powell M, Smith JW. Antibiol. Chemother. 1952; 2: 281.

2. Stephens VC, Conine JW. Antibiol. Ann. 1959; 346.

3. Paesen J, Roets E, Hoogmartens J. Chromatographia 1991; 32: 162.

4. Cachet Th, Lannoo P, Paesen J, Janssen G, Hoogmartens J. J. Chromatogr. 1992; 600: 99.

5. Cachet Th, Delrue M, Peasen J, Busson R, Roets E, Hoogmartens J. J. Pharm. Biomed. Anal. 1992; 10: 851 .

6. Olsen BA, Stafford JD, Reed DE. J. Chromatogr. 1992; 594: 203.

7. Shomo RE, Marshall AG. Int. J. Mass Spec. Ion Processes 1986; 72: 209.

8. Pleasance S, Kelly J, Leblanc MD, Quilliam MA, Boyd RK, Kitts DO, McErlane K, Bailey MR, North DH. Biol. Mass Spec. 1992; 21: 675 .

9. Shida Y, Deterding LJ, Hara KO, Kono M, Tomer KB. Tetrahedron 1993; 49: 9221.

10. Delelpine D, Hurtaud-Pessel D, Sanders P. J. AOAC Int. 1996; 79: 397.

11. Gates PJ, Kearney GC, Jones R, Leadlay PF, Staunton J. Rapid Commun. Mass Spectrom. 1999; 13: 242.

12. Volmer DA, Hui JPM. Rapid Commun. Mass Spectrom. 1998; 12: 123. 